\title{
Let the Machines Aware that they are Working Side by Side with Humans
}

\section{Hashim AYB*}

Faculty of Manufacturing Engineering, Department of Robotics and Automation, Universiti Teknikal, Malaysia

Keywords: Human's waveforms; Electromyography; Humanmachine interface; Self-awareness computing

\section{Introduction}

Ten or more years ago, the notion of human and machine interfaces was the field of much research and exploration. Testing and developing the system that was known as the human-machine interface (HMI) had been one of the popular trends where machine developers raced to implement the technology onto their system designs.

Now, the system developers are talking about machines that would have some degrees of awareness. In other words, the machines will have some built-in intelligence as the results of implementing self-awareness computing. One can readily find industrial robots that would reduce their motion's speed upon detecting the presence of human operators nearby. It is forecasted that there will be about two million units of an industrial robot in operational by the year 2017 [1]. By the same year, all industrial robots should expect to possess functional self-awareness modules.

Moreover, the concept of humanoid robots working side by side with humans has been gaining acceptance. It seems that humans have reached the height where they are acting gods that the machines are their creations. Instead of engineer the molecules; humans engineer the source codes that are highly complex that the machines' ability to become self-aware is evident.

There is massive junk of data generated, processed and shared in the World Wide Web (WWW). The machines would as well deal with it because they would typically be connected the cloud environment. Without this feature, the machines are categorized as outright obsolete. Depending on the level of tech-savvy, humans, also deal with Big Data as they tuned in. Differing from the machines, humans have the luxury of deciding when to get connected to the WWW or when to ignore it.

Since the human brain is a natural computer by itself, it also has massive information stored. One could never measure the Terabytes of data in the brain. However, one can surely scan the brain activities using one of the special techniques known as the magnetic resonance imaging (MRI). Linking the brain activities and Big Data has been an issue, not because the technology is non-existence, but the ethics of the decisions made based on the scan results [2]. Suppose the global cloud environment (GCE) represents the internet, we define the local cloud environment (LCE) as the network of a human brain. If we subscribe to the existence of the Akashic records [3] or the Lauh Mahfuz [4], then they represent the universal cloud environment. However, these two databases are not recognized by the secular scientific community. For the purpose of discussion in this paper, we only consider the global and the local cloud environments.

\section{Models}

The brain activity is about processing the data received from the bulk of somatosensory that collect data, according to the degree of sensations. The sensations are then converted into signals represented as myoelectricity [5]. The low-frequency $(0.5-100 \mathrm{~Hz})$ voltage waveforms are sent to the brain and are being processed, translated into descriptions of phenomena, are being stored that later may be retrieved [6]. All the information is said to exist in the LCE. Since humans are naturally intelligent, how they become even more sophisticated is due to training and learning of new knowledge.

On the other hand, machines must have some abilities to play humans, at least they can decide on some tasks. Since machines are synthetic entities, their ability to make decisions must lie in the application of a computer system [7]. They pick up information digitally, processed them into distinct digital formats that represent some descriptions of certain phenomena [8]. The processing techniques would have somewhat complicated procedures that allow them to come up with new information, be it originated from the GCE or LCE. One example of the processes may be found in the self-aware computing approach.

In Figure 1, the GCE shown at the top consists every module there is for a cloud system. For the humans and the machines to access the GCE, they will need to pass through an interface device through a local area network (LAN). Wire or wireless methods may make the connection. This is a multi-dimension data highway model between the humans and the machines. It suggests that machines will have the ability to let humans intercede with them.

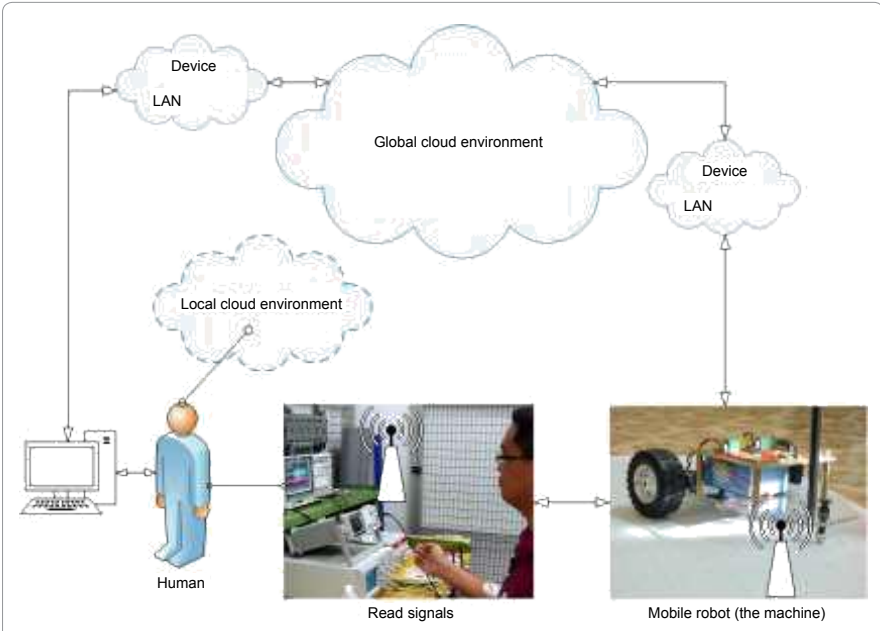

Figure 1: Surface electromyography.

${ }^{*}$ Corresponding author: Hashim AYB, Faculty of Manufacturing Engineering Department of Robotics and Automation, Universiti Teknikal, Malaysia, Tel: +606 331 6083; E-mail: yusairi@utem.edu.my

Received August 12, 2015; Accepted August 13, 2015; Published August 23 2015

Citation: Hashim AYB (2015) Let the Machines Aware that they are Working Side by Side with Humans. Adv Robot Autom 4: e126. doi:10.4172/2168-9695.1000e126

Copyright: ( 2015 Hashim AYB. This is an open-access article distributed under the terms of the Creative Commons Attribution License, which permits unrestricted use, distribution, and reproduction in any medium, provided the original author and source are credited. 
The LCE, nevertheless, is the human thinking. The environment is the "Self Big Data" where some of the information nodes may be private and personal. While other information nodes may be formal and are used to perform specific tasks such as the intention and the operation of a CNC machine. With these, the human operator knows what to do according to the prescribed standard operating procedures and he/she is certified fit for the job.

The machines, nevertheless, have embedded computer systems. Assume that the systems are high end and the programmer has uploaded very sophisticated algorithms, the machines should have now become entities that could make decisions and are self-aware. They also have the capabilities to link with the GCE.

Suppose we need to extract information from the LCE through the skin in the forearm. One of the most effective ways is to perform sEMG (surface electromyography) such as seen in Figure 1. The signals are read by attaching three electrodes on the skin that spot to a particular muscle. The biomechanics of the human body the function of muscles activities. For instance, the flexion carpus utalis muscle for an arm flex, or the extensor digitorium tendon for a hand close. On muscles activities, the signals that emanate from the skin is read by the transducer system. The patterns of the signals can be observed on an oscilloscope.

Figure 1 proposed model for multi-dimension data highway between the humans and the machines. The model suggests that machines will have the ability to let humans intercede with them. The kind of feeling that the machines have will allow humans and machines to work side by side.

Assume that one intends to bend the arm. So the intention to bend arm appears in the LCE. Now, the information needs to be realized by some myoelectric signals that should be sent to the respective muscles in the arm. Upon flexing, the transducer system reads the signals that can be seen and recorded using the oscilloscope. It is hypothesized that the signals contain information of "the intention", "the degree of the intention", "a unique biometric identification". The information belongs to an LCE, hence the person who carries it.
If the information package is linked to a machine, it may translate the package in several modes. It could be a discrete control mode where the information is treated as instructions. Alternatively, it could be read as values to be computed. Alternatively, it could be a handshaking input that the machine may identify the presence of a person listed in its database.

\section{Remarks}

We highlight the importance of humans and machines relationship to the level where the machines should no longer be treated as slaves but entities that are aware. The humans and the machines cannot escape the dynamics of massive data in the cloud environment, or they render themselves obsolete in the social networks. The data science of the environments and the kind of feeling that the machines have will allow humans and machines to work side by side in the future.

\section{Acknowledgment}

The Malaysian Ministry of Education supports this research through a research grant-FRGS/2/2013/SG02/FKP/02/2/F00176.

\section{References}

1. Brown AS (2015) Robots Grow in Numbers. Mech Eng 137: 32-33.

2. Smith N (2015) Interview - Marc Goodman. Eng Technol 3: 52-55

3. Todeschi KJ (1998) Edgar Cayce on the Akashic Records. ARE Press.

4. Frame JD (1921) Metaphysics and Cosmography in Persia Today. Muslim World 11: 272-280

5. Bani Hashim AY, Maslan MN, Izamshah R, Mohamad IS (2014) Delivering key signals to the machine: seeking the electric signal that muscles emanate. $\mathrm{J}$ Phys Conf Ser 546:1-8.

6. Genuth I (2015) Brain computer interfaces bring neuroscience to the masses Eng Technol 10: 37-39.

7. Bani Hashim AY, Fu Z, Jamaludin Z, Mohamad IS (2015) How Electromyography Readings from the Human Forearm are Made Cryptic, Trivial, or Non-Trivial Information for Use in Synthetic Systems. 5th International Conference on Biomedical Engineering and Technology 81: 6-10.

8. Fu Z, Bani Hashim AY, Jamaludin Z, Mohamad IS (2015) Decoding wrist gesture with combinational logic for the development of a practical EMG electrode sleeve. Mechanical Engineering Research Day 81-82. 